\title{
Joint Sink Mobility and Node Deployment for Prolonging Lifetime in Wireless Sensor Network
}

\author{
Huiyong Yuan \\ Department of Computer Science, Shaoguan University,Shaoguan Guangdong 512005, China; \\ email: hnldyhy@126.com
}

Keywords: Wireless sensor network; Node deployment; Mobile sink; Network lifetime

\begin{abstract}
When cluster heads transmit their data to the sink via multi-hop mode, the cluster heads closer to the sink are burdened with heavy relay traffic and tend to die early. In this paper, taking both sink mobility and node deployment into account, we investigate the energy-hole problem of data gathering in wireless sensor networks, and propose a new node deployment strategy for multi-hop wireless sensor networks with mobile sink. Simulation experiments show that the proposed strategy can effectively balance the sensors energy consumption and prolong the network lifetime.
\end{abstract}

\section{Introduction}

With the development of the MEMS (micro-electro-mechanical-systems), memory technologies and recent advances in microprocessor and wireless communication technologies, it's possible to produce micro sensors[1]. For some applications (such as forest monitoring ), a large-scale sensor network can be deployed, with thousands or more sensors densely distributed in the interested area and working cooperatively for data collection and transmission.

Due to the limited computing power, sensing range, and transmission range of individual sensors, the sensor network is formed to detect the indicated phenomenon and to deliver the collected data to the sink via multiple hops. In order to prolong network lifetime, the sensors can be organized hierarchically by grouping them into clusters. In clustered wireless sensor networks, the sensors do not transmit their collected data to the sink, but to designated cluster heads which aggregate the data packets and send them to the sink via multiple hops.

For multi-hop mode, the cluster heads closest to the sink are burdened with a heavy relay traffic load and die first [2]. In case of sensors failure or malfunctioning around the sink, the sensor network connectivity and coverage may not be guaranteed. No matter how many remaining sensors are still active, none of them can communicate with the sink. As a result, the system lifetime becomes short.

There are a lot of interests of introducing sink mobility into wireless sensor networks for lifetime improvement. With a mobile sink, the cluster heads around the sink always changes, thus balancing the energy consumption in the entire network and improving the network lifetime[3].

Deployment of sensors in a wireless sensor network is a critical task as deployment should be optimum to increase network lifetime [4]. In most current designs, random and uniform deployment is popular proposed schemes due to their simplicity. However, the traditional random and uniform deployments are not suitable because of the energy-hole problem.

In this paper, taking both sink mobility and node deployment into account, we investigate the energy-hole problem of data gathering in wireless sensor networks, and proposed a new node deployment strategy for multi-hop wireless sensor networks. Simulation experiments show that the proposed strategy can effectively balance the sensors energy consumption and prolong the network lifetime. 


\section{Related Work}

Much work has been done during recent years to increase the lifetime of sensor networks. Node deployment has received considerable attention recently. Yuh-Ren Tsai [5] focuses on the node deployment problem for large-scale randomly distributed wireless sensor networks, and propose a non-uniform sensor deployment strategy for the multi-hop routing. In [6], the authors exhibit the weakness of the uniform distribution by disclosing the fatal sink routing-hole problem, and propose power-aware sensor deployment scheme based on a general sensor application model. The authors of [7] conclude that in a circular multi-hop sensor network with non-uniform sensor distribution, the unbalanced energy consumption among all the sensors in the network is unavoidable, and propose a non-uniform sensor distribution strategy to achieve nearly balanced energy consumption in the network. However, all these proposals assume that sensor networks are modeled with only static sink, i.e., sink do not have mobility.

In order to tack the unbalanced energy depletion and extend the network lifetime, other introduce mobility into the WSNs. The authors of [8] study the maximum lifetime problem of wireless sensor networks where a mobile sink can visit only small number of locations. The sensors near the sink would change overtime with a sink moving in the network, thus mitigating the energy imbalance around the sink. In [3] , the authors show that the network lifetime can be extended significantly if the mobile sink moves around the periphery of sensor networks. They assume that, if the mobile sink can balance the traffic load of the sensors, the lifetime of the network can increase. Therefore, they propose an optimization problem for choosing a mobility strategy that minimizes the maximum traffic load of the sensors.

The authors of [9] make use of a mobile relay to prolong the network lifetime. They state that the mobile relay only needs to stay with in two hops away from the sink to enhance the network lifetime by a factor of nearly four. They also propose two joint mobility and routing algorithms capable of attaining the claimed results.

Our work differs from the above works since we take both sink mobility and node deployment into account. We first derive the optimal cluster radius and then propose a new node deployment strategy for wireless sensor network with mobile sink.

\section{System Model and Formulation}

We assume there is an energy-efficient MAC protocol in the underlying MAC layer, energy will be consumed only when performing sensing task, processing raw data, and transmitting and receiving data for itself and other sensors. The radio model discussed in [10] can be used to evaluate energy consumption of data transmission. In this model, a radio dissipates $E_{\text {elec }}$, defined for the transmitter or receiver circuitry, and $E_{a m p}$, defined for the transmitter amplifier.

We assume all sensors have transmit power control and can use just the minimum required energy to send information to the recipients. The equations used to model energy consumption of a sensor for communication are given below.The energy consumption for transmitting sensor:

$$
E_{T X}(\text { len }, d)=E_{\text {elec }} \times \text { len }+E_{\text {amp }} \times \text { len } \times d^{2}
$$

The energy consumption for receiving sensor:

$$
E_{R X}(\text { len }, d)=E_{\text {elec }} \times \text { len }
$$

Here $d$ is the distance between two sensors, len is the number of bits of information sent, and $E_{\text {elec }}$ and $E_{\text {amp }}$ are the constants as previously defined.

The energy dissipation is a second order function of distance. So the data routing with multiple shorter nearby hops will be more efficient than directly transmitting between two far sensors. The energy consumption is also a linear function of len which is bits of information transmitted through the sensor network.

We assume that $\mathrm{N}$ sensors are deployed in a rectangular $2 W \times W$ observation region. The movement trajectory of the mobile sink is on concentric of the rectangular, because it is the only 
symmetric strategy that we can have within the network region. The mobile sink collects data from cluster heads. We also assume that all sensors have the same initial energy Einit and the energy of the mobile sink is unlimited. Each sensor generates a raw message packet with the same size len-bits. Sensor network is organized into clusters whose radius is equal to r. Cluster heads can aggregate their members data into single-sized data packet and forward it to the mobile sink. Fig.1 depicts an application where sensors periodically transmit their data to the sink. The figure illustrates that cluster heads transmit the aggregated data in multi-hop mode.

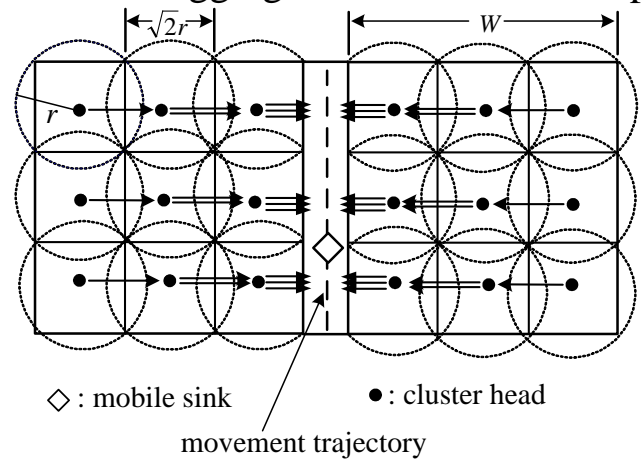

Fig.1. Mobile sink network model

To facilitate our discussion, we divide sensors to different sets according to their distance to the mobile sink. The set $P_{i}$ contains all sensors which can reach the mobile sink with minimal hop count $i$. The sensor $S_{j}$ will be in the set $P_{i}$ if $(i-1) \times \sqrt{2} r<\operatorname{dist}\left(S_{j}, \sin k\right) \leq i \times \sqrt{2} r$, where $\operatorname{dist}\left(S_{j}\right.$, sink $)$ is the Euclidean distance between sensor $S_{j}$ and the mobile sink. Thus, the sensors in set $P_{i}$ will be in the $i$-th cell to the movement trajectory of the mobile sink.

In our network model, the sensors at the same cell will die at almost the same time. Maximizing the network lifetime is equivalent to minimizing the average energy consumption of the sensors. We also know that an optimal deployment should have an equal lifetime for all sensors in the network. The lifetime $T_{i}$ of sensors in set $P_{i}$ is defined as the expected time for the battery energy $E_{\text {init }}$ to be exhausted, that is, $T_{i}=E_{\text {init }} / E_{i}$, where $E_{i}$ is the average energy consump-tion per round. We define network lifetime $T_{\text {network }}$ of a sensor network as the minimum lifetime of all sensors in the network, that is

$$
T_{\text {network }}=\min \left\{T_{i}\right\}
$$

Based on equations (3), the maximum network lifetime problem can be writen as follows

$$
\begin{array}{ll}
\text { Maximize } & T_{\text {network }} \equiv \min \left\{T_{i}\right\} \\
\text { Subject to } & T_{i}=E_{\text {init }} / E_{i_{-} \text {average }}, 1 \leq i \leq k \\
& k=\left\lceil\frac{W}{\sqrt{2} r}\right\rceil
\end{array}
$$

Our objective is to find the optimal cluster radius $r$ and the number of sensors in set $P_{i}$.

\section{Solutions for the Problem}

Mhatre and Rosenberg in [11] define characteristic distance as that distance which when used as the inter-sensor distance, minimizes the energy consumption for sending a data packet from a source sensor to a destination sensor. This characteristic distance, $d_{\text {char }}$, is

$$
d_{\text {char }}=\sqrt{2 E_{\text {elec }} / E_{\text {amp }}}
$$

Only width $d_{\text {char }}$ can the energy consumption rate be minimized. A good clustering algorithm may average the workload on each sensor and the generated clusters should have the same cluster sizes. Thus, we set the optimal cluster radius $r=d_{\text {char }}$.

In this section, we first study the energy consumption. Let us consider a single cluster with radius $r$. We assume that $N_{\mathrm{k}}$ denotes the number of sensors in set $P_{\mathrm{k}}$. The average number of sensors in a cluster is 


$$
N_{k \_c l u}=N_{k} / W \times \sqrt{2} r
$$

To keep the total energy dissipation within the cluster as small as possible, the cluster head should be positioned at the centroid of the cluster area $A$. In this case, the square of distance between cluster members and the cluster head is given as:

$$
d_{\text {to_cluster }}^{2}=\iint_{A}\left(x^{2}+y^{2}\right) d x d y=r^{2} / 2
$$

The transmitter energy consumption of cluster members in set $P_{\mathrm{k}}$ is given by

$$
E_{k \_ \text {member }}=\operatorname{len}\left(N_{k \_c l u}-1\right)\left(E_{\text {elec }}+E_{\text {amp }} d_{\text {to_cluster }}^{2}\right)
$$

where we denote len as the length of the data packet.

Each cluster head receives the data from all member nodes in the cluster, aggregates and transmits to the sink. The energy required is

$$
E_{k_{\_} \text {cluster }}=\operatorname{len}\left(N_{k_{-} c l u}-1\right) E_{\text {elec }}+\operatorname{len} E_{\text {amp }}(\sqrt{2} r)^{2}
$$

Thus, the average energy consumption of set $P_{\mathrm{k}}$ in a round is

$$
E_{k \_ \text {average }}=\left(E_{k_{-} \text {member }}+E_{k_{-} \text {cluster }}\right) / N_{k_{-} \text {clu }}
$$

We assume that $N_{\mathrm{i}}$ denotes the number of sensors in set $P_{\mathrm{i}}$. Let us consider the average energy consumption of set $P_{\mathrm{i}}$ in a round. The average number in a cluster of set $P_{\mathrm{i}}$ is

$$
N_{i_{-} \text {clu }}=N_{i} / W \times \sqrt{2} r
$$

The transmitter energy consumption of cluster members in a cluster of set $P_{\mathrm{i}}$ is

$$
E_{i \_m e m b e r}=l e n\left(N_{i \_c l u}-1\right)\left(E_{\text {elec }}+E_{\text {amp }} d_{\text {to_cluster }}^{2}\right)
$$

The energy depletion of cluster head for receiving data is given by

$$
E_{i_{\_} \text {cluster_rec }}=\operatorname{len}\left(N_{i_{-} \text {clu }}-1\right) E_{\text {elec }}+\operatorname{len}(k-i) E_{\text {elec }}
$$

The energy consumption for transmitting data to set $P_{\mathrm{i}-1}$ is given by

$$
E_{i \_c l u s t e r \text { trans }}=\operatorname{len}(k-i+1)\left(E_{\text {elec }}+E_{\text {amp }} \times(\sqrt{2} r)^{2}\right)
$$

Thus, the average energy consumption of set $P_{\mathrm{i}}$ in a round is

$$
E_{\text {i_average }}=\left(E_{i \_m e m b e r}+E_{i \_c l u s t e r \_r e c}+E_{i \_c l u s t e r \_t r a n s}\right) / N_{i \_c l u}
$$

To fully utilize total energy allocated to all sensors in the deployment region and avoid energy waste, we want to ensure that all sensors have the same lifetime, which is equal to the network lifetime, $T_{\text {net }}$. Consequently, the number of sensors that resides in $P_{\mathrm{i}}$, denoted by $N_{\mathrm{i}}$, can calculated by solving $E_{i \_a v e r a g e}=E_{k \_ \text {average }}$.

The total number of nodes deployed in the network is given by

$$
N=\sum_{i=1}^{k} N_{i}
$$

Clustering a wireless sensor network means partitioning its sensors into clusters, each one with a cluster head and some sensors as its members. 


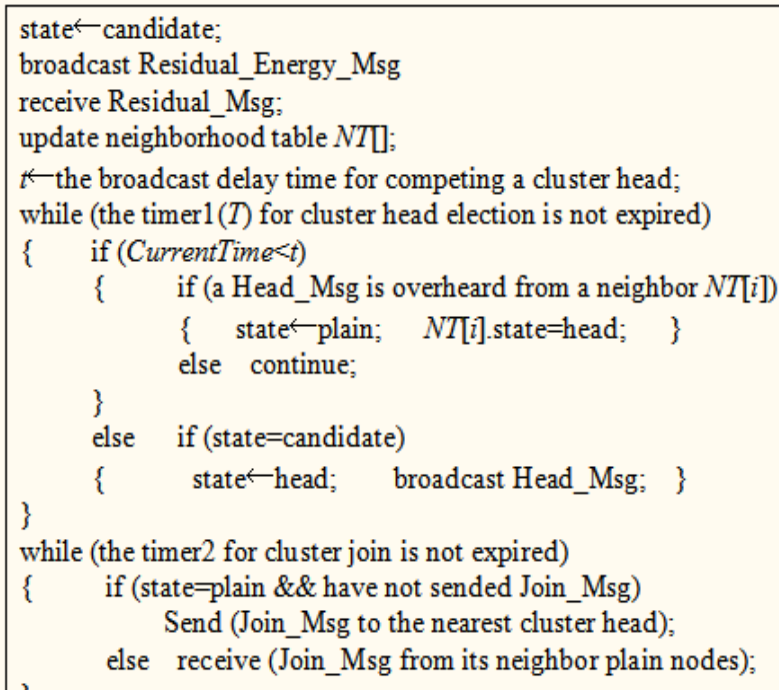

Fig.2. Cluster head selection pseudocode

Our algorithm is a distributed cluster heads competitive algorithm, here cluster head selection is primarily based on the residual energy and position of each sensor. Every sensor become a tentative cluster head with the same probability p which is a predefined threshold. The algorithm pseudocode for an arbitrary sensor $\mathrm{i}$ is given in Fig. 2.

\section{Simulation and Results}

In this section, we conduct extensive simulations to evaluate the performances of our proposed node deployment strategy and compared it with random deployment. We use the same energy consumption model as [10]. Every result shown below is the average of 100 independent experiments.

We first compare the sensors lifetime of our proposed strategy and random deployment. As shown in Fig.3, in random deployment, the lifetime of a sensor decreases with the decreasing distance between the mobile sink and sensor. However, in our proposed node deployment strategy, different sensor densities are assigned to different cells to balance the energy depletion of each cell. Thus, our proposed strategy can ensure that all sensors have almost same lifetime, which is equal to the network lifetime.

We then observe the effect of network region size on the network lifetime. In this experiment, we compare the network lifetime with varied $W$ from 50 to 200 meters. To deploy sensors with relatively high density, there are 25 to 400 sensors.

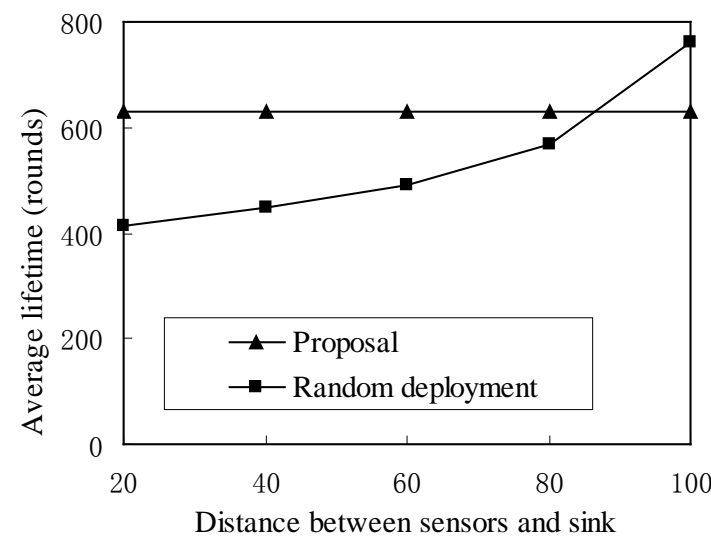

Fig.3. Sensors lifetime comparison.

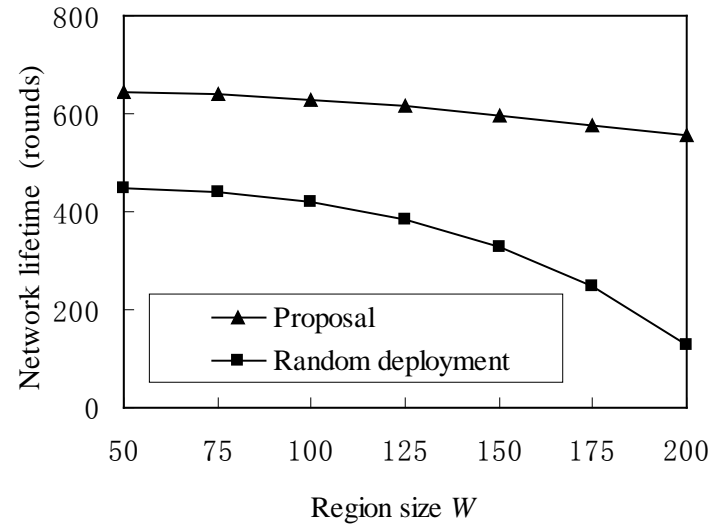

Fig.4. Network lifetime comparison

Fig. 4 shows the effect of different network region sizes on the network lifetime of two strategies. It can be seen that network region size does not have great impact on network lifetime 
of our proposed strategy, but the network lifetime of uniform distribution drops sharply as the network region size expends. Therefore, our proposed strategy leads to a better performance in term of scalability.

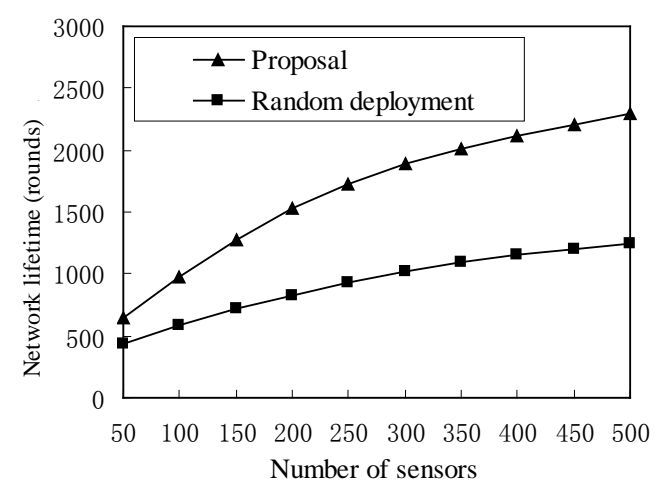

Fig.5. Network lifetime comparison with different sensor density

Finally, we study the impact of sensor density on the network lifetime. Fig.5 shows the comparison of network lifetime as we set the number of sensors at 100 and 500, using 50 sensors spacing. The network lifetime is the number of rounds until the first sensor dies. As expected, network lifetime using our proposed strategy is efficiently prolonged compared with random deployment.

\section{Conclusion}

In this paper, we investigate the energy-hole problem of data gathering in wireless sensor networks, and proposed a node deployment strategy for wireless sensor networks with mobile sink. Simulation experiments show that the proposed strategy can effectively balance the energy consumption and prolong the network lifetime.

\section{Acknowledgement}

This research has been supported by the science and technology project of Shaoguan (Grant NO.2012 CX/K91) and Science Foundation of Hunan Province (Grant No. 11JJ3074).

\section{References}

[1] IF. Akyildiz, W.Su, Y. Sankarasubramaniam, E.Cayirci, Wireless sensor networks: A survey, Comput[C]. Netw, 2002.

[2] S. Olariu and I. Stojmenovic. Design guidelines for maximizing lifetime and avoiding energy holes in sensor networks with uniform distribution and uniform reporting[C]. In proceedings of INFOCOM, 2006.

[3] J. Luo and J. Hubaux. Joint mobility and routing for lifetime elongation in wireless sensor networks[C]. Proc. Of the 24th Annual Conference of the IEEE Computer and Communication Societies , 2005.

[4] Sha Chao,Wang Ru-chuan. Energy-efficient node deployment strategy for wireless sensor networks[J].The Journal of China Universities of Posts and Telecommunications, 2013:19(10): 110-120.

[5] Yuh-Ren Tsai, Kai-Jie Yang , Sz-Yi Yeh. Non-uniform node deployment for lifetime extension in large-scale randomly distributed wireless sensor Networks[C], In proceedings of AINA,2012.

[6] Y. Liu, H. Ngan , L. M. Ni. Power-aware node deployment in wireless sensor networks[C]. In proceedings of IJDSN, 2007. 
[7] G. Chen, S. K.Das , X. Wu. Avoiding energy holes in wireless sensor networks with nonuniform node distribution[J]. IEEE Trans. On Parallel and Distributed System, 2008(10):710-720.

[8] I. Papadimitriou , L.Georgiadi. Maximum lifetime routing to mobile sink in wireless sensor networks[C], in the 13th IEEE SoftCom, 2005.

[9] W.Wang, V.Srinivasan, K.C.Chua. Using mobile relays to prolong the lifetime of wireless sensor networks[C]. In Proc. Of the 11th ACM MobiCom,2005.

[10] W.R.Heinzelman, A.Chandrakasan. An application-specific protocol architecture for wireless microsensor networks[J]. IEEE Transactions on Wireless Communications, 2002(4):660-669.

[11] V. Mhatre, C. Rosenberg. Design guidelines for wireless sensor networks: communication, clustering and aggregation[J]. Ad Hoc Networks Journal, 2004(1):45-63. 